\title{
Application of an optical clearing agent during noninvasive laser coagulation of the canine vas deferens
}

\author{
Christopher M. Cilip \\ University of North Carolina at Charlotte \\ Department of Physics and Optical Science \\ 9201 University City Boulevard \\ Charlotte, North Carolina 28223
}

\author{
Ashley E. Ross \\ Jonathan P. Jarow \\ Johns Hopkins Medical Institutions \\ Department of Urology \\ 601 North Caroline Street \\ Baltimore, Maryland 21287
}

\author{
Nathaniel M. Fried \\ University of North Carolina at Charlotte \\ Department of Physics and Optical Science \\ 9210 University City Boulevard \\ Charlotte, North Carolina 28223 \\ and \\ Johns Hopkins Medical Institutions \\ Department of Urology \\ 601 North Caroline Street \\ Baltimore, Maryland 21287
}

\begin{abstract}
Development of a noninvasive vasectomy technique may eliminate male fear of complications and result in a more popular procedure. This study explores application of an optical clearing agent (OCA) to scrotal skin to reduce laser power necessary for successful noninvasive laser vasectomy and eliminate scrotal skin burns. A mixture of dimethyl sulfoxide and glycerol was noninvasively delivered into scrotal skin using a pneumatic jet device. Near-infrared laser radiation was delivered in conjunction with cryogen spray cooling to the skin surface in a canine model, ex vivo and in vivo. Burst pressure (BP) measurements were conducted to quantify strength of vas closure. A 30-min application of OCA improved skin transparency by $26 \pm 3 \%$, reducing average power necessary for successful noninvasive laser vasectomy from $9.2 \mathrm{~W}$ without OCA (BP $=291 \pm 31 \mathrm{mmHg})$ to $7.0 \mathrm{~W}$ with OCA $(B P=292 \pm 19 \mathrm{mmHg})$. Control studies without OCA at $7.0 \mathrm{~W}$ failed to coagulate the vas with burst pressures $(82 \pm 28 \mathrm{mmHg})$ significantly below typical ejaculation pressures $(136 \pm 29 \mathrm{mmHg})$. Application of an OCA reduced the laser power necessary for successful noninvasive thermal coagulation of the vas by $\sim 25 \%$. This technique may result in use of a less expensive laser and eliminate the formation of scrotal skin burns during the procedure. $\odot 2010$ Society of Photo-Optical Instrumentation Engineers. [DOI: $10.1117 / 1.3463009$ ]
\end{abstract}

Keywords: laser; vasectomy; male sterilization; noninvasive; incisionless; optical clearing.

Paper 10092PR received Feb. 23, 2010; revised manuscript received May 18, 2010; accepted for publication May 21, 2010; published online Jul. 21, 2010.

\section{Introduction}

Approximately 500,000 vasectomies are performed in the United States per year making it the most common urological procedure in the U.S. ${ }^{1}$ Although more effective and less likely to have complications than tubal ligation, the number of men undergoing surgical sterilization is approximately threefold less than women. ${ }^{2-4}$ Fear of complications related to surgical vasectomy (e.g., hematoma, infection, acute and chronic pain, sterilization failure) remains a prominent factor. ${ }^{5,6}$ The availability of a rapid, cost-effective, straightforward, and noninvasive technique for male sterilization is likely to improve the popularity of vasectomy.

Our laboratory is currently developing a noninvasive vasectomy technique utilizing near-infrared laser irradiation in conjunction with cryogen spray cooling of the scrotal skin surface for thermal occlusion of the vas deferens. During previous studies, we have reported successful targeting, thermal occlusion, and scarring of the vas while minimizing scrotal skin injury in ex vivo and in vivo canine models. ${ }^{7,8}$

Optical clearing agents (OCA) have been explored over the past decade with great interest by numerous investigators

Address all correspondence to: Nathaniel M. Fried, Ph.D., Department of Physics and Optical Science, University of North Carolina at Charlotte, 9201 University City Boulevard, Charlotte, NC 28223. Tel: 704-687-8149; Fax: 704-6878197; E-mail: nmfried@uncc.edu for reducing light scattering in skin and increasing the optical transmission through skin for both therapeutic and diagnostic light-based procedures. ${ }^{9-27}$ Although the mechanism of optical clearing of skin is still being studied, the basic effect is understood. Common skin components, hydrated collagen $(n=1.42)$, stratum corneum $(n=1.55)$, and interstitial fluid $(n=1.36)$ have different refractive indices. As the interstitial fluid content in the skin is reduced due to diffusion driven by the osmotic stress induced by the OCA (e.g., glycerol with an $n=1.47$ ), skin components become more closely packed and form a more homogenous medium. As the OCA diffuses into the tissue, it further matches the tissue matrix, leading to a significant reduction in light scattering and a corresponding increase in optical transmission. ${ }^{14}$

Common OCAs include dimethyl sulfoxide (DMSO), glycerol, glucose, and other sugar compounds. In this study, we used a DMSO/glycerol mixture, taking advantage of the excellent transport properties of DMSO while minimizing its concentration due to potential toxicity concerns, and relying on glycerol for optical clearing of the scrotal skin.

The Madajet, a commercially available device currently marketed for noninvasive delivery of local anesthesia through the scrotal skin during conventional no-scalpel vasectomy, was used in this study for delivery of the OCA. The Madajet

1083-3668/2010/15(4)/048001/6/\$25.00 @ $2010 \mathrm{SPIE}$ 
provides a compact, inexpensive alternative method of temporarily breaching the stratum corneum compared to previously reported mechanical and optical methods (e.g., needle injection, tape stripping, sandpaper, microneedle rollers, flashlamp irradiation of carbon dots, and laser ablation). ${ }^{13-15,17,18,22,23,26,27}$ The Madajet also allows delivery of significantly less OCA $(0.4 \mathrm{ml})$ than conventional syringe needle injection $(4 \mathrm{ml})$, thus reducing the potential risks of tissue toxicity from DMSO. (This data were acquired in our laboratory during preliminary studies using a graduated cylinder to measure the quantity of OCA delivered by Madajet versus syringe needle.)

The purpose of this study is to explore the use of an OCA to increase the transparency of the scrotal skin, thus reducing the laser power necessary for successful thermal coagulation of the canine vas deferens and to completely eliminate the formation of minor scrotal skin burns, observed during previous studies.

\section{Methods}

\subsection{Optical Clearing Agent}

A 1:3 ratio of DMSO (Gaylord Chemical, Slidell, Louisiana) and glycerol (Fisher Scientific, Pittsburgh, Pennsylvania) was used as an OCA. DMSO was chosen because of its transport properties in tissue. However, the concentration of DMSO used was minimized due to concerns for potential toxicity. Glycerol was chosen because it is biocompatible and has been previously shown to increase skin transparency. The refractive index of our OCA (1:3 ratio of DMSO/glycerol) was determined to be $n=1.51$ by measuring the angle of deviation of the collimated ytterbium fiber laser beam $(\lambda=1075 \mathrm{~nm})$ using a hollow prism with and without the liquid present, as previously described. $^{28}$

The OCA was delivered to the skin using a pneumatic jet device (Madajet, Advanced Meditech International, Flushing, New York) normally used for noninvasive delivery of anesthesia during the conventional no-scalpel vasectomy procedure [Fig. 1(a)]. A total of four sites on the skin surface were treated with the OCA, corresponding to a diamond configuration immediately bordering the targeted vas area [Fig. 1(b)]. Each of the four sites received $0.1 \mathrm{ml}$ of the OCA in the same 1:3 DMSO/glycerol ratio, resulting in a total of $0.4 \mathrm{ml}$ delivered to the skin per a vas. The skin was then allowed to sit for $30 \mathrm{~min}$, providing sufficient time for the OCA to diffuse into the treatment site and increase the transparency of the scrotal skin. The treatment site was not directly treated with the Madajet, to avoid unnecessary tissue swelling, which had been observed to interfere with the delivery path of the laser radiation to the underlying vas during preliminary studies.

\subsection{Ex Vivo Tissue Studies}

Scrotal skin and vas tissue were harvested from male dogs immediately after sacrifice for unrelated experiments. The tissue was then partially submerged in a temperature-controlled saline bath, placed on a hotplate, and maintained at $\sim 37^{\circ} \mathrm{C}$. A standard 4.0-mm-ID vasectomy clamp (VE-1, Advanced Meditech, Flushing, New York), custom coated in our laboratory with an FEP Teflon ${ }^{\circledR}$ tubing for thermal insulation, was then used to tightly grasp the vas and surrounding scrotal skin, using a previously reported method. ${ }^{7}$

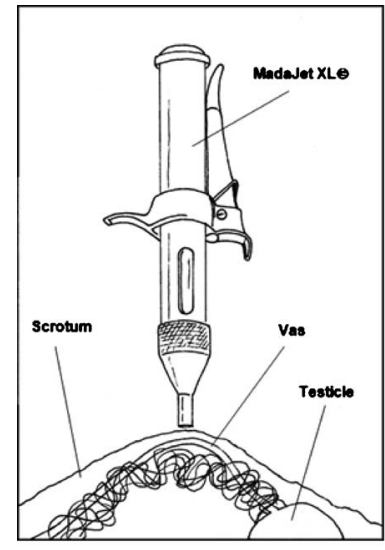

(a)

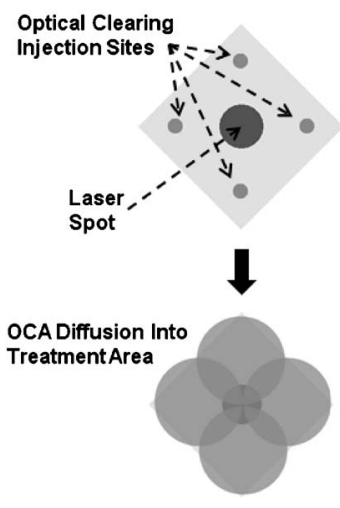

(b)
Fig. 1 (a) Madajet (pneumatic jet device) used for noninvasive delivery of small quantities of the OCA through the skin. (Reproduced with permission from Pfenninger Medical Procedures Center P.C., John L. Pfenninger, M.D.). (b) Pattern for delivering the OCA on the treatment site. Four injections were spaced equally around the 3-mm-diam laser spot, and then the OCA was allowed to diffuse into the laser treatment area over time.

\subsection{In Vivo Animal Studies}

All procedures were conducted under an animal protocol approved by the Johns Hopkins Animal Review Committee. Noninvasive thermal occlusion of the vas was performed bilaterally in a total of $5 \mathrm{dogs}(n=10$ vasa). The study was divided into two groups. In group 1, noninvasive laser vasectomy was performed at an average power of $9.2 \mathrm{~W}$ and cryogen cooling rate of $0.25 \mathrm{~Hz}$ without application of an OCA. In group 2, noninvasive laser vasectomy was also performed but at an average power of $7.0 \mathrm{~W}$ and cryogen cooling rate of $0.20 \mathrm{~Hz}$ with application of an OCA. These parameters were chosen based on the success of previous ex vivo tissue studies. Figure 2 shows the experimental setup for the in vivo canine studies. For both groups, after completion of the procedure, the vas tissue was harvested and burst pressure (BP) measurements were performed. All of the dogs used for this study were neutered immediately after the procedure and then adopted out to caring homes.

\subsection{Laser Parameters}

A compact, tabletop, $50 \mathrm{~W}$ continuous-wave ytterbium fiber laser (Model No. TLR1075-50, IPG Photonics, Oxford, Massachusetts) emitted radiation with a wavelength of $1075 \mathrm{~nm}$ that was then focused with a $300-\mathrm{mm}-\mathrm{FL}$ lens into a $400-\mu \mathrm{m}$ silica fiber-optic patch cord. A lens at the end of the fiber-optic patch cord delivered a collimated laser beam to the tissue surface (Fig. 2). A function generator was used to electronically modulate the fiber laser for delivery of the laser radiation in pulsed mode, producing an average output power of 7.0-9.2 W, 500-ms pulse duration, $0.5 \mathrm{~Hz}$ pulse rate, and 3-mm-diam spot at the scrotal skin surface.

\subsection{Cooling Parameters}

A dynamic cooling device (Candela Laser Corporation, Wayland, Massachusetts) was used to deliver the cryogen (halocarbon 134a, 1,1,1,2-tetrafluoroethane, boiling point= 


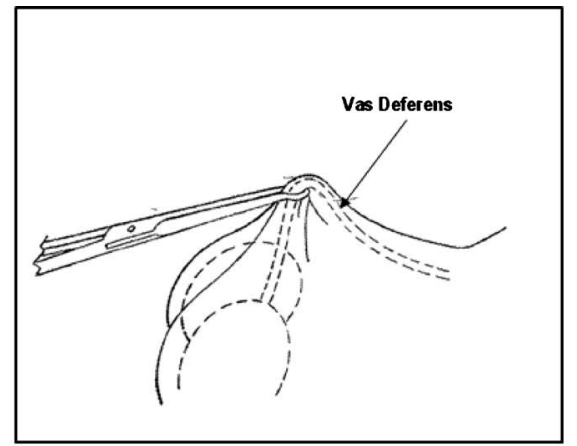

(a)

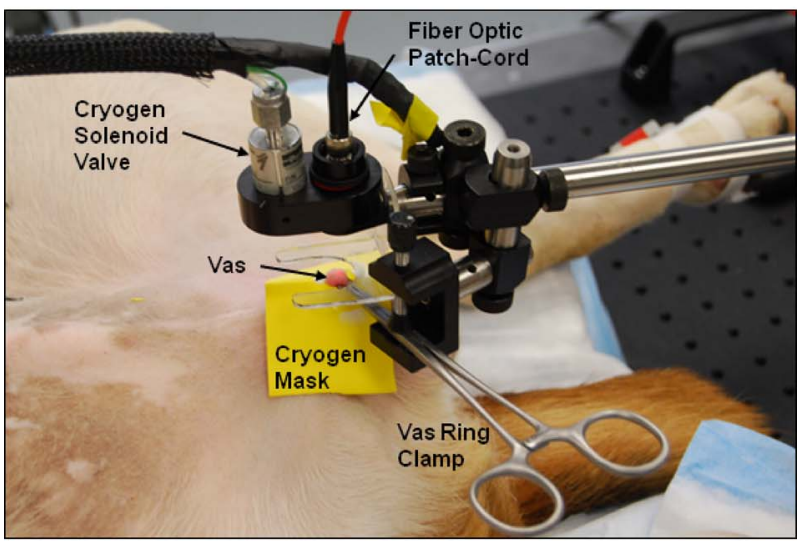

(b)

Fig. 2 (a) Diagram of the vas and vas ring clamp. (b) Photograph of the experimental setup for noninvasive laser vasectomy in dogs, in vivo.

$-26{ }^{\circ} \mathrm{C}$ ) to the tissue surface through a solenoid valve (Fig. 2 ). The solenoid valve was externally triggered using a function generator and an oscilloscope to view the pulse characteristics. Three cryogen pulses were applied to precool the scrotal skin surface prior to laser irradiation. During irradiation, the cryogen spray was delivered intermittently between laser pulses with a $60-\mathrm{ms}$ pulse duration, pulse rate of $0.20-0.25 \mathrm{~Hz}$, and a 2-cm-diam spot concentric with the laser spot. A cryogen mask made of a yellow rubber material was used to thermally insulate the surrounding scrotal skin from the cryogen spray and hence avoid superficial freeze burns observed during previous studies. ${ }^{8}$

\subsection{Indicators of Vas Occlusion}

Vas BP measurements were performed to quantify the degree of closure of the thermally coagulated vas (Fig. 3). A threeway stopcock was connected to a $50 \mathrm{ml}$ syringe, pressure transducer, and hypodermic needle. The syringe was filled with saline, which was also distributed throughout the entire system. The vas was attached to a $27 \mathrm{G}$ hypodermic needle and clamped with hemostats. A pressure analyzer unit was calibrated to zero setting. Saline from the syringe was then slowly pumped into the lumen of the vas using a syringe pump at a rate of $0.01 \mathrm{ml} / \mathrm{s}$, resulting in an elevated pressure until the vas burst open.

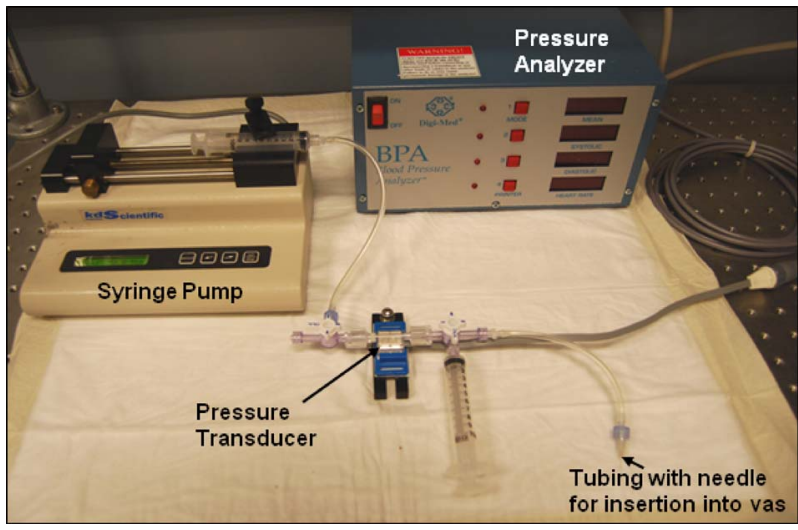

Fig. 3 Photograph of experimental setup for measuring vas BP, consisting of a pressure transducer, pressure analyzer unit, and syringe pump.

\section{Results}

\subsection{Transmission Studies}

Transmission studies performed in canine scrotal skin samples, ex vivo, demonstrated that the application of the OCA (DMSO/glycerol) for $30 \mathrm{~min}$ improved skin transparency by an average of $26 \pm 3 \%$, as shown in Fig. 4. These values were used in calculating the reduction in laser power necessary for use in the noninvasive laser vasectomy studies. It should be noted that although optical transmission for the DMSO/glycerol mixture continued to increase beyond $30 \mathrm{~min}$, these studies were not extended beyond this time range because of concern that longer surgical preparation times would probably not be suitable for vasectomy, which is already a relatively brief procedure.

\subsection{Noninvasive Laser Vasectomy Studies}

$\mathrm{BP}$ results as a function of the laser/cooling parameters for both the ex vivo and in vivo noninvasive laser vasectomy procedures performed in this study, as well as in previously reported studies, ${ }^{7,8}$ are compared in Table 1 . The average power necessary for successful noninvasive laser vasectomy was re-

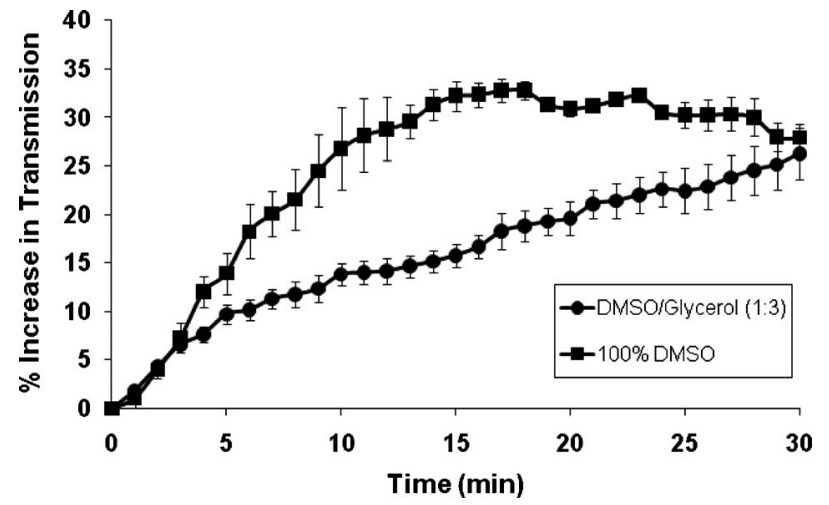

Fig. 4 Optical transmission studies performed in canine scrotal skin, ex vivo, after Madajet delivery of an OCA composed of either $100 \%$ DMSO or a 1:3 ratio of DMSO and glycerol. Percent increase in optical transmission is plotted as a function of time. The average and standard deviation was plotted for each data set ( $n=4$ samples). 
Cilip et al.: Application of an optical clearing agent during noninvasive laser coagulation...

Table 1 Comparison of BP results for ex vivo and in vivo noninvasive laser vasectomy studies performed with and without an OCA.

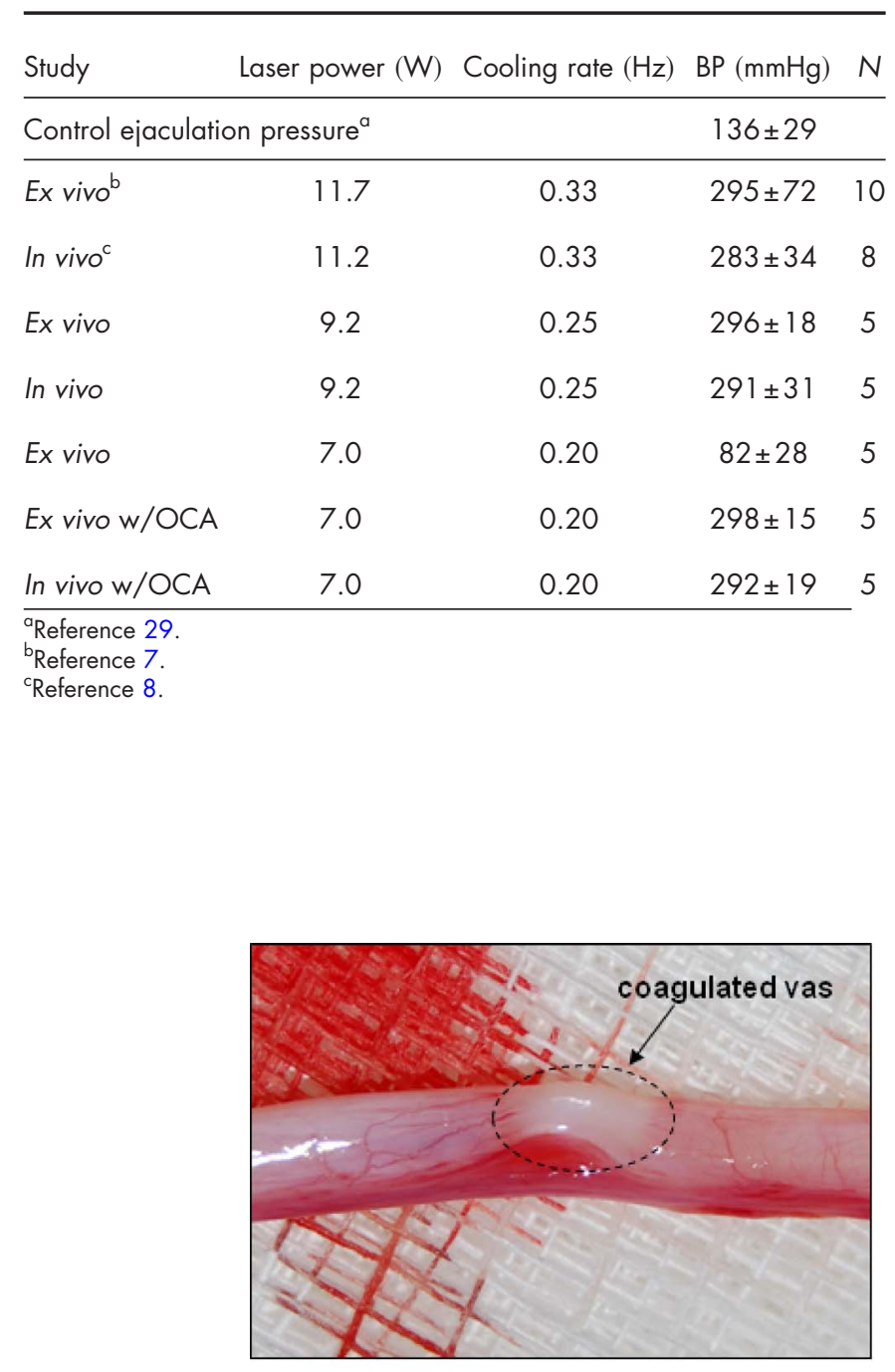

(a)

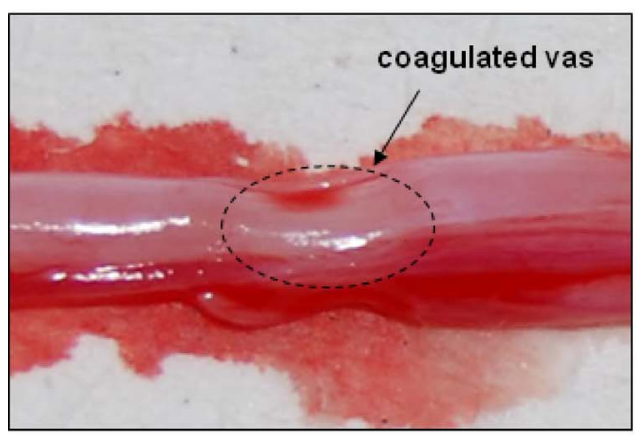

(c) duced from $9.2 \mathrm{~W}$ without $\mathrm{OCA}(\mathrm{BP}=291 \pm 31 \mathrm{mmHg})$ to $7.0 \mathrm{~W}$ with OCA $(\mathrm{BP}=292 \pm 19 \mathrm{mmHg})$. Control studies without $\mathrm{OCA}$ at $7.0 \mathrm{~W}$ failed to coagulate the vas with $\mathrm{BP}$ $(82 \pm 28 \mathrm{mmHg})$ significantly below typical ejaculation pressures $(136 \pm 29 \mathrm{mmHg}){ }^{29}$ These lower power settings also eliminated the formation of minor scrotal skin burns previously observed using higher average powers to the tissue. ${ }^{8}$

Images of the canine vas and scrotal skin taken immediately after the in vivo noninvasive laser vasectomy procedure are shown in Fig. 5. Thermally coagulated sections of the canine vas demonstrated characteristic signatures of blanching and shrinkage along an $\sim 3$-mm-long segment, corresponding to the laser spot diameter [Figs. 5(a) and 5(c)]. Grossly, thermal effects were similar when the vas was coagulated using 9.2 W, $0.25 \mathrm{~Hz}$ without OCA or when $7.0 \mathrm{~W}, 0.20 \mathrm{~Hz}$, with OCA were used [Figs 5(c) and 5(a), respectively]. Figures 5(b) and 5(d) show the scrotal skin surface in the region of the procedure both with and without the use of OCA and low power, respectively. In Fig. 5(b), the blanched area corresponds to where the OCA was applied, and the two compression marks correspond to where the vas ring clamp was closed down on the scrotal fold $(7.0 \mathrm{~W}, 0.20 \mathrm{~Hz}$, with OCA). In comparison to Fig. 5(d), which shows the scrotal skin surface after the procedure without OCA, the scrotal skin after use of

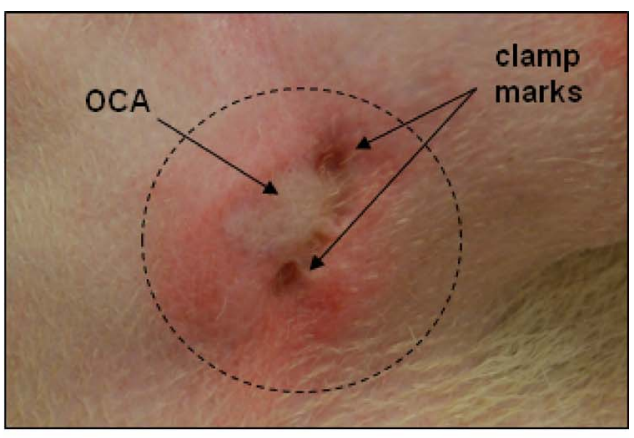

(b)

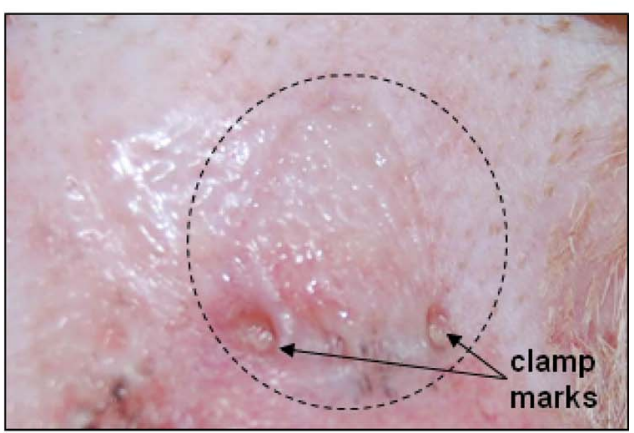

(d)

Fig. 5 Gross analysis of vas and scrotal skin conducted immediately after noninvasive laser vasectomy procedure on dogs, in vivo. (a) Thermally coagulated section of canine vas (dotted circle) demonstrating characteristic signatures of blanching and shrinkage along an $\sim 3$-mm-long segment corresponding to laser spot diameter $(7.0 \mathrm{~W}, 0.20 \mathrm{~Hz}$, with OCA). (b) Scrotal skin surface in region of procedure (dotted circle). Swollen area corresponds to where OCA was applied. Two compression marks correspond to where vas ring clamp was applied (7.0 W, 0.20 Hz, with OCA). (c) Thermally coagulated section of canine vas (dotted circle) demonstrating characteristic signatures of blanching and shrinkage along an $\sim 3$-mm-long segment corresponding to laser spot diameter $(9.2 \mathrm{~W}, 0.25 \mathrm{~Hz}$, without OCA). (d) Scrotal skin surface after procedure without OCA $(9.2 \mathrm{~W}, 0.25 \mathrm{~Hz}$, without OCA). Note that image (b) shows more tissue trauma than (d), in part, due to the need to leave vas ring clamp on skin for prolonged period of time as OCA needed $30 \mathrm{~min}$ to diffuse into skin. This could easily be eliminated in a human model where vas is much easier to grasp. 
OCA [Fig. 5(b)] appeared to have more acute tissue trauma. This was due, in part, to the need to leave the vas ring clamp on the skin for a prolonged period of time because the OCA needed 30 min to diffuse into the skin. This complication may be eliminated in a human model, where the vas is much easier to grasp and the OCA timing and vas surgical preparation can be more easily coordinated.

\section{Discussion}

Vasectomy is a safe, simple, effective, and inexpensive surgical procedure for male sterilization. Despite the low morbidity of this procedure, societal pressures and male fear of surgery are reasons frequently cited by those choosing other forms of contraception. The development of a completely noninvasive laser vasectomy technique may eliminate these concerns and increase male acceptance of vasectomy.

Our laboratory is currently developing a noninvasive vasectomy technique utilizing near-infrared laser irradiation in conjunction with cryogen spray cooling of the scrotal skin surface for thermal occlusion of the vas deferens. During previous studies, we have reported successful targeting, thermal occlusion, and scarring of the vas while minimizing scrotal skin injury in an ex vivo and in vivo canine model. ${ }^{7,8}$

Higher laser power is associated with increased local tissue trauma. Indeed, in this study, by optimizing our laser parameters, we were able to decrease the power setting (from 11.2 to $9.2 \mathrm{~W}$ ) with equivalent or superior occlusive results to our previous studies while minimizing skin injury. ${ }^{8}$ In addition, this study demonstrates that it is possible to decrease the average power even further to $7 \mathrm{~W}$ if the procedure is combined with noninvasive delivery of an OCA to the scrotal skin, which increases skin transparency. Using this technique, the vas was consistently thermally coagulated and occluded as indicated by the high vas BP measurements, and indicators of minor scrotal skin burns observed during previous procedures were also completely eliminated.

Although the noninvasive aspect of our laser vasectomy procedure remains the primary attraction of this technique, cost is an important factor as well. It should be noted that the cost of surgical lasers usually scales with output power. Thus, any approach that results in a net reduction in laser power for noninvasive vasectomy may directly translate into a less expensive procedure. This is important because the current, conventional no-scalpel vasectomy procedure is already a short, inexpensive procedure.

\section{Conclusions}

Application of an OCA reduced the average power necessary for successful noninvasive laser coagulation of the vas deferens by $\sim 25 \%$. This technique may result in the use of a less expensive laser system and eliminate the formation of scrotal skin burns during the procedure.

\section{Acknowledgments}

This project is supported by a research grant from the NIH, National Institute of Child Health and Human Development, and the U.S. Agency for International Development, through a subcontract from Family Health International, Durham, North Carolina. The authors thank Christina Crothers, Dawn Ruben, and Laurie Pipitone for assisting with the animal studies, and
James Hsia of the Candela Corporation (Wayland, Massachusetts) for providing the cryogen cooling system used in these studies.

\section{References}

1. M. A. Barone, P. L. Hutchinson, C. H. Johnson, J. Hsia, and J. Wheeler, "Vasectomy in the United States, 2002," J. Urol. 176, 232 236 (2006).

2. W. D. Mosher, G. M. Martinez, A. Chandra, J. C. Abma, and S. J. Wilson, "Use of contraception and use of family planning services in the United States: 1982-2002," Adv. Data 350, 1-36 (2004).

3. A. Chandra, G. M. Martinez, W. D. Mosher, J. C. Abma, and C. Jones, "Fertility, family planning, and reproductive health of U.S women: data from the 2002 National Survey of Family Growth," Vital Health Stat. 25, 1-160 (2005).

4. G. M. Martinez, A. Chandra, J. C. Abma, J. Jones, and W. D. Mosher, "Fertility, contraception, and fatherhood: data on men and women from cycle 6 (2002) of the 2002 National Survey of Family Growth," Vital Health Stat. 26, 1-142 (2006).

5. W. B. Miller, R. N. Shain, and D. J. Pasta, "Tubal sterilization or vasectomy: how do married couples make the choice," Fertil. Steril. 56, 278-284 (1991).

6. R. N. Shain, W. B. Miller, and A. E. Holden, "Factors associated with married women's selection of tubal sterilization and vasectomy," Fertil. Steril. 43, 234-244 (1985).

7. C. M. Cilip, J. P. Jarow, and N. M. Fried, "Noninvasive laser vasectomy: preliminary ex vivo tissue studies," Lasers Surg. Med. 41, 203-207 (2009).

8. C. M. Cilip, A. E. Ross, J. P. Jarow, and N. M. Fried, "Noninvasive laser coagulation of the canine vas deferens, in vivo," Proc. SPIE 7548, 75481D (2010).

9. G. Vargas, E. K. Chan, J. K. Barton, H. G. Rylander, 3rd, and A. J. Welch, "Use of an agent to reduce scattering in skin," Lasers Surg. Med. 24, 133-141 (1999).

10. G. Vargas, K. F. Chan, S. L. Thomsen, and A. J. Welch, "Use of osmotically active agents to alter optical properties of tissue: effects on the detected fluorescence signal measured through skin," Lasers Surg. Med. 29, 213-220 (2001).

11. X. Xu and R. K. Wang, "Synergistic effect of hyperosmotic agents of dimethyl sulfoxide and glycerol on optical clearing of gastric tissue studied with near infrared spectroscopy," Phys. Med. Biol. 49, 457468 (2004).

12. B. Choi, L. Tsu, E. Chen, T. S. Ishak, S. M. Iskandar, S. Chess, and J. S. Nelson, "Determination of chemical agent optical clearing potential using in vitro human skin," Lasers Surg. Med. 36, 72-75 (2005).

13. R. J. McNichols, M. A. Fox, A. Gowda, S. Tuya, B. Bell, and M. Motamedi, "Temporary dermal scatter reduction: quantitative assessment and implications for improved laser tattoo removal," Lasers Surg. Med. 36, 289-296 (2005).

14. O. F. Stumpp, A. J. Welch, T. E. Milner, and J. Neev, "Enhancement of transepidermal skin clearing agent delivery using a $980 \mathrm{~nm}$ diode laser," Lasers Surg. Med. 37, 278-285 (2005).

15. G. Altshuler, M. Smirnov, and I. Yaroslavsky, "Lattice of optical islets: a novel treatment modality in photomedicine," J. Appl. Phys., J. Phys. D 38, 2732-2747 (2005).

16. J. Hirshburg, B. Choi, J. S. Nelson, and A. T. Yeh, "Collagen solubility correlates with skin optical clearing," J. Biomed. Opt. 11, 040501 (2006).

17. O. Stumpp, B. Chen, and A. J. Welch, "Using sandpaper for noninvasive transepidermal optical skin clearing agent delivery," $J$. Biomed. Opt. 11, 041118 (2006).

18. V. V. Tuchin, G. B. Altshuler, A. A. Gavrilova, A. B. Pravdin, D. Tabatadze, J. Childs, and I. V. Yaroslavsky, "Optical clearing of skin using flashlamp-induced enhancement of epidermal permeability," Lasers Surg. Med. 38, 824-836 (2006).

19. V. V. Tuchin, "A clear vision for laser diagnostics (review)," IEEE J. Sel. Top. Quantum Electron. 13, 1621-1628 (2007).

20. M. Kinnunen, R. Myllyla, and S. Vainio, "Detecting glucose-induced changes in in vitro and in vivo experiments with optical coherence tomography," J. Biomed. Opt. 13, 021111 (2008).

21. Z. Mao, D. Zhu, Y. Hu, X. Wen, and Z. Han, "Influence of alcohols on the optical clearing effect of skin in vitro," J. Biomed. Opt. 13, 021104 (2008). 
22. J. Yoon, T. Son, E.-H. Choi, B. Choi, J. S. Nelson, and B. Jung, "Enhancement of optical skin clearing efficacy using a microneedle roller," J. Biomed. Opt. 13, 021103 (2008).

23. E. A. Genina, A. N. Bashkatov, A. A. Korobko, E. A. Zubkova, V. V. Tuchin, I. V. Yaroslavsky, and G. B. Altshuler, "Optical clearing of human skin: comparative study of permeability and dehydration of intact and photothermally perforated skin," J. Biomed. Opt. 13, 021102 (2008).

24. A. K. Bui, R. A. McClure, J. Chang, C. Stoianovici, J. Hirshburg, A. T. Yeh, and B. Choi, "Revisiting optical clearing with dimethyl sulfoxide (DMSO)," Lasers Surg. Med. 41, 142-148 (2009).

25. M. Zimmerley, R. A. McClure, B. Choi, and E. O. Potma, "Following dimethyl sulfoxide skin optical clearing dynamics with quantita- tive nonlinear multimodal microscopy," Appl. Opt. 48, D79-D87 (2009).

26. A. N. Bashkatov, E. A. Genina, V. V. Tuchin, and G. B. Altshuler, "Skin optical clearing for improvement of laser tattoo removal," $\mathrm{La}$ ser Phys. 19, 1312-1322 (2009).

27. C. Liu, Z. Zhi, V. V. Tuchin, Q. Luo, and D. Zhu, "Enhancement of skin optical clearing efficacy using photo-irradiation," Lasers Surg. Med. 42, 132-140 (2010).

28. M. D. Edmiston, R. Van Fossen Ramsey, and N. Szczepanski, "A liquid prism for refractive index studies," J. Chem. Educ. 78, 1479$1480(2001)$

29. A. Shafik, "Electrovasogram: a canine study of the electromechanical activity of the vas deferens," Urology 46, 692-696 (1995). 